\title{
Retina Vascular Structures near the Optic Disc and in the Macula in Primary Angle-Closure Suspects
}

\author{
Xiaohua Pana,b Merry Z.C. Ruanc Wudi Fan ${ }^{c, d}$ Kai Cao ${ }^{a, e}$ Hui Feng ${ }^{a}$ \\ Zhongyin $\mathrm{Hu}^{f}$ Yue Yang ${ }^{g}$ Fancheng Yan ${ }^{\mathrm{h}}$ Mengya Zhao ${ }^{\mathrm{i}}$ Qian Liu ${ }^{j}$ Min Fu $^{k}$ \\ Fei Xiang ${ }^{\text {a }}$ Richard Leel Shuning $\mathrm{Li}^{\mathrm{a}}$ Ying Han ${ }^{\mathrm{c}, \mathrm{m}}$ \\ aDepartment of Ophthalmology, Beijing Tongren Eye Center, Beijing Tongren Hospital, Beijing, China; ${ }^{\text {bShenzhen }}$ \\ Eye Hospital, Jinan University, Shenzhen Eye Institute, Shenzhen, China; 'Department of Ophthalmology, University \\ of California San Francisco, San Francisco, CA, USA; dCollege of Engineering, University of California, Berkeley, CA, \\ USA; ${ }^{B}$ Beijing Institute of Ophthalmology, Beijing Tongren Eye Center, Beijing Tongren Hospital, Capital Medical \\ University, Beijing, China; 'Department of Ophthalmology, First People's Hospital of Yunnan Province, Kunming, \\ China; ${ }^{9}$ Department of Ophthalmology, The Third Hospital of Mianyang, Sichuan Mental Health Center, Mianyang, \\ China; ' Department of Ophthalmology, Beijing Friendship Hospital, Capital Medical University, Beijing, China; \\ 'Department of Ophthalmology, Shanghai General Hospital, Shanghai, China; ${ }^{j}$ Henan Eye Institute, Henan Eye \\ Hospital, Henan Provincial People's Hospital and Zhengzhou University People's Hospital, Zhengzhou, China; \\ kDepartment of Ophthalmology, Zhujiang Hospital, Southern Medical University, Guangzhou, China; 'Bascom \\ Palmer Eye Institute, University of Miami Miller School of Medicine, Miami, FL, USA; mophthalmology Section, \\ Surgical Service, San Francisco Veterans Affairs Medical Center, San Francisco, CA, USA
}

\section{Keywords}

Vessel density · Fovea avascular zone - Optical coherence tomography angiography · Primary angle-closure suspect

\begin{abstract}
Introduction: The main aim of this article was to study the retinal peripapillary and macular vascular structures in eyes with primary angle-closure suspects (PACS) using optical coherence tomography angiography (OCTA). Methods: In this cross-sectional study, control and PACS subjects were recruited from a community screening. Only one eye per subject was used for analysis. All participants underwent a questionnaire survey, physical and ophthalmic examinations, ocular biometry measurements, and OCTA. We compared basic demographics and vessel structure parameters between control and PACS eyes. Univariate and multivariate
\end{abstract}

linear regression analyses were performed to investigate factors associated with vascular parameters in both groups. Results: Data from 254 subjects including 155 PACS and 99 controls were analyzed. In the peripapillary region, PACS eyes showed similar retina nerve fiber layer (RNFL) and vessel densities (VDs) including and excluding large vessels compared to control eyes. Compared to control eyes, all macular OCTA parameters showed significant differences in PACS eyes, including decreased superficial VD $(p=0.006)$ and deep VD ( $p=0.004)$, larger fovea avascular zone (FAZ) area $(p=0.006)$, and longer FAZ perimeter $(p=0.004)$. Gen$\operatorname{der}(p=0.039)$, age $(p<0.001)$, and Garway-Heath superior hemisphere RNFL $(p<0.001)$ were risk factors influencing optic disc VD excluding large vessels. Axial length was the

Xiaohua Pan, Merry Z.C. Ruan, and Wudi Fan were listed as co-first authors and contributed equally to the manuscript.
(C) 2021 The Author(s)

Published by S. Karger AG, Basel

This is an Open Access article licensed under the Creative Commons Attribution-NonCommercial-4.0 International License (CC BY-NC) (http://www.karger.com/Services/OpenAccessLicense), applicable to the online version of the article only. Usage and distribution for commercial purposes requires written permission.
Correspondence to:

Shuning Li, lishuningqd@163.com

Ying Han, ying.han@ucsf.edu 
major factor affecting macula superficial and deep VDs ( $p=$ 0.004 and 0.001 respectively), while PACS was an independent factor associated with larger FAZ perimeter $(p=0.046)$. Conclusion: While PACS and control eyes have comparable RNFL and vascular structure around the optic nerve head, macular vascular structures are significantly different.

(c) 2021 The Author(s).

Published by S. Karger AG, Basel

\section{Introduction}

Primary angle-closure disease (PACD) is a group of ocular diseases in whichmore than 180 degrees of the posterior pigmented trabecular meshwork is not visible on static gonioscopy with or without glaucomatous changes [1]. PACD is the leading cause of irreversible blindness in Asia [2]. In contrast to primary angle-closure glaucoma (PACG), in which optic nerve damage occurs, primary angle-closure suspect (PACS) is benign but the most common type of PACD.

Since PACS patients do not exhibit optic neuropathy and generally have normal intraocular pressures (IOP) during clinic visits, one would assume there are no differences in macular and optic nerve vascular structures between PACS and healthy eyes. On the other hand, it is unclear whether PACS patients experience intermittent IOP elevation or heightened diurnal IOP change. Moreover, whether PACG progresses from PACS remains controversial $[3,4]$. Since vascular structure changes can be early signs of glaucomatous optic neuropathy and visual field loss [5], examining and reporting the vascular changes in patients with PACS is important.

Optical coherence tomography angiography (OCTA) has emerged as a noninvasive imaging technique which provides visualization of fundus structure and the vascular microcirculation [6-8]. The optic disc and macula are two important structures in the posterior segment of the eye. Studies have shown the high repeatability between intra-visit, inter-visit, and interobserver in measuring vessel density (VD) and foveal avascular zone $[9,10]$. A growing number of studies show correlations between vascular structures and the progression of glaucomatous optic neuropathy and visual field loss [11-13]. However, this has not been explored in patients with PACS.

In this study, we will describe vascular changes in patients with PACS by examining blood flow and structural features around the optic disc and the macular using OCTA and comparing them with normal subjects. In addition, we will investigate potential factors influencing vascular structures in control and PACS eyes.

\section{Materials and Methods}

\section{Study Population}

All subjects in this cross-sectional study were Han Chinese 50 years or older recruited from Daxing District, Beijing, China. Patients were identified through a community screening. During the screening, interviewers obtained informed consent and conducted an interview on-site before patients proceeded to clinical assessments from October through November 2018. The study was approved by the Ethics Committee of Clinical Research at Beijing Tongren Hospital of Capital Medical University (approval number: TRECKY2019-071) and adheres to the Tenets of the Declaration of Helsinki. This study was registered with the Chinese Clinical Trial Registry (Registration Number: ChiCTR2000037944).

\section{Clinical Examination}

After initial recruitment, study participants visited community hospital eye centers for clinical assessments. Participants first completed questionnaires and interviews to provide their medical and ocular histories. After completing a basic physical examination and blood tests, participants received ophthalmic examinations and imaging. These tests included, but were not limited to, best corrected visual acuity measured by Snellen charts then converted to log scale, IOP, gonioscopy, slit lamp and fundus exam, biometry, and optic nerve OCT and OCTA (RNFL; Optovue Inc., Fremont, CA, USA). The IOP was measured by a non-contact tonometer without anesthesia (Topcon CT-80, Tokyo, Japan) after vision and refraction examination. The biometry was measured with a non-contact measurement device, Lenstar LS 900 (LENSTAR, LS900; Haag Streit, Bern, Switzerland). The IOP and ocular biometry parameters were measured three times for each eye, and the average value was used for statistical analysis. Gonioscopy (Ocular Technology Inc., Goleta, CA, USA) is a contact examination performed by an experienced glaucoma specialists (HY and LSN) in a darkroom. After topical anesthesia, static examination was performed with a narrow,1-mm-high light beam away from the pupil without indentation on the eye at the primary position of gaze. Dynamic examination was then performed by indentation to identify peripheral anterior synechia (PAS).

\section{Inclusion Criteria and Exclusion Criteria}

Inclusion criteria for patients with PACS included: age $\geq 50$ years old, a minimum of 180 degrees of iridotrabecular contact with nonvisualization of the posterior trabecular meshwork on static gonioscopy, absence of PAS, IOP $\leq 21 \mathrm{~mm} \mathrm{Hg}$, absence of glaucomatous optic neuropathy (defined as vertical cup-to-disc ratio $[\mathrm{C}: \mathrm{D}] \leq 0.6$ and between eye vertical C:D $<0.2$, no optic disc hemorrhages, no optic nerve rim loss or notch, and no retinal nerve fiber layer defects on spectral-domain ocular coherence topography). Patients meeting any of the following criteria were excluded: presence of additional ocular pathology, prior iris laser treatment or intraocular surgery, history of intraocular inflammation, trauma, congenital malformation, secondary angle-closure, prior episode of acute primary angle closure in either eye, or refractive error greater than +3.00 diopters or less than -6.00 diopters. Control subjects had normal eye exam and open-angle structures by gonioscopy, and also met exclusion criteria above. In addition, all participants with diabetes mellitus (hemoglobin A1C > 6.4) were excluded from the study [14]. Only the right eye of each qualifying participant was included in the study. 
Optical Coherence Tomography Angiography

OCTA images were acquired with tracking mode. The axial resolution of each scan is $5 \mu \mathrm{m}$. 4.5 by $4.5 \mathrm{~mm}$ scans of optic disc images and 6 by $6 \mathrm{~mm}$ scans of retina images were captured for each qualified eye in Figure 1. While fovea avascular zone (FAZ) parameters are measured on $3 \times 3$-mm OCTA images in most studies, we performed the study with $6 \times 6$-mm scans to obtain boarder macular area. Dong et al. [15] reported that no statistically significant differences were found in FAZ between the two scan sizes. The scan was performed by experienced technicians who were masked from other exam findings. Images with an overall quality index $>6$ without severe artifacts were analyzed with the AngioVue software RTVue XR Avanti System Version 2017.1 (Optovue; Optovue, Inc., Fremont, CA, USA). VD was represented by pixels with decorrelation values above the threshold. The boundary of the radial peripapillary capillary (RPC) was from the inner limiting membrane to the posterior surface of RNFL. Larger vessels $(>32 \mu \mathrm{m})$ were excluded automatically to compute capillary densities within the all-plexus slab to effectively remove the projection artifacts of larger vessel slab on deeper vascular slab.

For optic disc image analyses, the peripapillary region was defined as the 4 -mm-diameter area excluding the central 2 -mm-diameter circle centered on the optic. AngioVue analysis automatically segmented the peripapillary region into 8 Garway-Heath $(\mathrm{GH})$ segments (nasal superior, nasal inferior, inferior nasal, inferior temporal, temporal inferior, temporal superior, superior temporal, superior nasal). Optic disc margin was automatically detected on Bruch's membrane opening. The optic cup is defined by internal limiting membrane segmentation line crossing below the Bruch's membrane opening plane.

For macular analyses, the VD was measured through an ETDRS grid comprised of 3 concentric rings: $1 \mathrm{~mm}$ center (fovea), 1-3 mm (parafovea), and an outer ring of 3-6 mm diameters (perifovea). Angiography analyses were segmented into 4 layers: (1) macula superficial vascular layer: internal limiting membrane to $10 \mu \mathrm{m}$ above inner plexiform layer; (2) deep vascular layer: $10 \mu \mathrm{m}$ above IPL to 10 $\mu \mathrm{m}$ below outer plexiform layer; (3) outer retina: $10 \mu \mathrm{m}$ below OPL to $10 \mu \mathrm{m}$ above Bruch's membrane, and (4) choroid: $10 \mu \mathrm{m}$ above BRM to $40 \mu \mathrm{m}$ below BRM. Data collected included FAZ area, FAZ perimeter (PERIM), acircularity index, and flow density, which excludes the effect of vessel thickness on OCTA measurements.

\section{Correction of the Ocular Magnification Effect}

Studies have proposed that the magnification of the imaging device and the magnification of the eye result in the magnification of the OCTA fundus image. While various correction methods have been proposed, Garway-Heath et al. [16] concluded that the single most accurate correction method is to correct for the magnification error using the axial length. Thus, we used the axial length data combined with the Littmann's method and the Bennett's formula to calculate the actual size of ocular parameters. The Littmann formula is expressed as $D_{t}=p \times q \times D_{m}$, where $D_{t}$ denotes the true distance, $D_{m}$ denotes the measured distance, $p$ denotes the magnification due to the imaging system, and $\mathrm{q}$ denotes the magnification due to the eye [17]. The magnification due to the eye $(q)$ is calculated with the Bennett formula of $q=0.01306 \times(\mathrm{AL}$ - 1.82). For our imaging device, the axial length at which the true distance is equal to the measured distance $\left(D_{t}=D_{m}\right)$ is $23.95 \mathrm{~mm}$. This value allows us to calculate the $p$ value with the Bennett formula, as $\mathrm{D}_{\mathrm{t}}=\mathrm{D}_{\mathrm{m}}$ implies $\mathrm{p} \times \mathrm{q}=1$. Therefore, $p=1 / \mathrm{q}=1 /(0.01306$ $\times[23.95-1.82])=3.46$. Therefore, the formula we employ to cor-
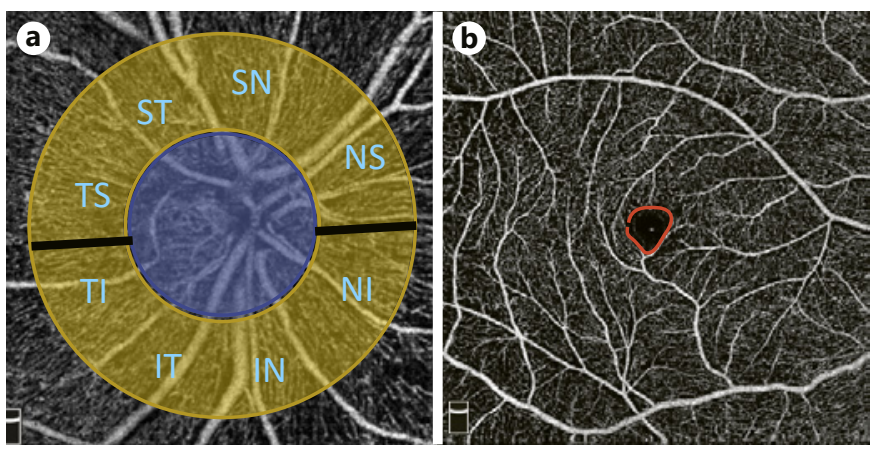

Fig. 1. Illustration of the areas measured near optic disc and in the macula by OCTA. a Example of OCTA scan near the optic nerve. The dimension of the image is $4.5 \mathrm{~mm}^{2}$. For peripapillary RNFL and VD, the whole image was measured. For GH segment measurements, the optic nerve is marked in blue (circle diameter $=2$ $\mathrm{mm}$ ). GH segments are shown in yellow with green labels (donut diameter $=4 \mathrm{~mm}$ ). Superior hemisphere includes nasal superior (NS), temporal superior (TS), superior temporal (ST), and superior nasal (SN). Inferior hemisphere includes nasal inferior (NI), inferior nasal (IN), inferior temporal (IT), and temporal inferior (TI). $\mathbf{b}$ Example of OCTA scan of the macula. The dimension of the image is $6 \mathrm{~mm}^{2}$. Vessel densities were measured in the area shown. Center black area highlighted by red border is the FAZ.

rect for the ocular magnification effect is as follows: $D_{t}=p \times q \times$ $\mathrm{D}_{\mathrm{m}}=(3.46) \times(0.01306 \times[\mathrm{AL}-1.82]) \times \mathrm{D}_{\mathrm{m}}$.

\section{Statistical Analysis}

Data normality was verified with the Shapiro test. The median values and interquartile range were used for basic statistical description of non-normally distributed continuous variables, otherwise mean values with standardized deviations were used for statistical analysis. To compare differences between control and PACS group, $\chi^{2}$ tests were employed. In addition, all pairwise comparisons were performed among the VD in different GH sectors of PACS subjects with $p$ values adjusted using the Bonferroni method, if needed. Univariate analysis by linear mixed-effects modeling was used to determine the relevant factors of RNFL and VD. Indicators of statistical significance were included in the final multivariate analysis. Considering that colinearity existed among RNFL in different GH sectors, LASSO regression analyses were used and a proper RNFL was chosen. Considering the effect of signal strength index (SSI) on VD measurement, we included the SSI into the univariate and multivariate analysis of optic disc and macula VD [18, 19]. R (https://www.r-project.org/, version 4.0.0) was used for all data analyses. $p<0.05$ was considered statistically significant.

\section{Results}

\section{Study Cohort}

Eight hundred and nineteen subjects were originally interviewed for the study. Three hundred and fifty-eight 
Table 1. Demographic and clinical characteristics of the study population

\begin{tabular}{lllr}
\hline Patient information* & Control & PACS & $p$ value \\
\hline $\begin{array}{lll}\text { Patients, } n \\
\quad \text { Sex, } n \text { (\%) }\end{array} \quad 155$ & 99 & \\
$\quad$ Male & & & 0.292 \\
$\quad$ Female & $108(30.3)$ & $24(24.2)$ & \\
Average age & $59(55-65)$ & $75(75.8)$ & 0.148 \\
Systolic blood pressure & $139(132-150)$ & $134(127-147)$ & 0.060 \\
Diastolic blood pressure & $84(79-92)$ & $81(74-89)$ & 0.099 \\
Body mass index & $27.12(25.08-29.38)$ & $26.06(24.31-28.77)$ & 0.127 \\
Hemoglobin A1C & $5.7(5.3-6.2)$ & $5.6(5.1-6.0)$ & 0.270 \\
Best corrected visual acuity (LogMAR) & $0.10(0.00-0.22)$ & $0.10(0.00-0.10)$ & 0.883 \\
IOP & $16(15-18)$ & $16(15-18)$ & 0.540 \\
C:D & $0.36(0.00-0.48)$ & $0.31(0.00-0.51)$ & 0.447 \\
Central cornea thickness & $526 \pm 33$ & $527 \pm 28$ & 0.746 \\
Anterior chamber depth & $2.57 \pm 0.28$ & $2.20 \pm 0.25$ & $<\mathbf{0 . 0 0 1}$ \\
Lens thickness & $4.42(4.21-4.68)$ & $4.66(4.50-4.80)$ & $<\mathbf{0 . 0 0 1}$ \\
Axial length & $23.04 \pm 0.78$ & $22.60 \pm 0.69$ & $<\mathbf{0 . 0 0 1}$ \\
Spherical refractive error & $0.25(-0.50$ to 1.00) & $1.00(0.38-1.50)$ & $<\mathbf{0 . 0 0 1}$ \\
\hline
\end{tabular}

PACS, primary angle-closure suspect; IOP, intraocular pressure; C:D; cup-to-disk ratio. * Data that does not fit normal distribution are expressed as median (25th percentile to 75 th percentile). Data that fits normal distribution are expressed as mean \pm standard deviation. subjects were excluded from the analysis due to high hemoglobin A1C. Other subjects were excluded from the study due to diagnosis of PAC (16 subjects) or PACG (17 subjects), enlarged C:D (55 subjects), or quality index $\leq 6$ in OCTA imaging (119 subjects). Ninety-nine PACS subjects and 155 control subjects meeting inclusion and exclusion criteria were recruited into the study. The control and PACS populations are comparable in terms of gender, age, systolic blood pressure, diastolic blood pressure, BMI, A1C, best corrected visual acuity, C:D, IOP, and central cornea thickness. Compared to control eyes, PACS eyes exhibited features consistent with narrow angles including shallow anterior chamber depth (PACS vs. control: $2.20 \mathrm{~mm} \pm 0.25 \mathrm{vs} .2 .57 \mathrm{~mm} \pm 0.2$, average \pm standard deviation, $p<0.001$ ), short axial length (PACS vs. control: $22.60 \mathrm{~mm} \pm 0.69$ vs. $23.04 \mathrm{~mm} \pm 0.78, p<0.001$ ), and hyperopia (PACS vs. control: $+1.00 \mathrm{D}[+0.38 \mathrm{D}$ to $+1.50 \mathrm{D}]$ vs. $+0.25 \mathrm{D}[-0.50$ to +1.00$], p<0.001)$. Detailed demographics data are described in Table 1.

\section{Optic Disc Findings}

We compared optic disc RNFL thickness and VD including large vessels and excluding large vessels between control and PACS eyes (Table 2). No differences between RNFL thickness in the area $4.5 \mathrm{~mm}$ peripheral to the optic disc and with GH segment between the two groups were observed. In addition, we did not identify differenc- es in VD including or excluding big vessels near the optic disc (Table 2).

To investigate the potential factors that correlate with optic disc VD for all subjects, we performed univariate and multivariate analyses to explore potential risk factors, including demographic characters, basic physical exam and ophthalmic exam findings, biometry data, SSI of OCTA, and RNFL. In univariate analysis, we observed that gender (regression coefficient $\beta=-0.260, p<0.001$ ), age $(\beta=-0.413, p<0.001)$, systolic and diastolic blood pressure $(\beta=-0.182, p=0.004$ and $\beta=-0.129, p=0.040)$, C:D $(\beta=-0.212, p=0.001)$, SSI $(\beta=0.267, p<0.001)$, and superior and inferior RNFL $(\beta=0.590, p<0.001$ and $\beta=$ $0.573, p<0.001$ ) were related to optic disk VD excluding large vessels. In multivariate analysis, our data supported that female sex (beta regression coefficient $\beta=-0.597$, $p=0.039)$, younger age $(\beta=-0.114, p<0.001)$, or thicker superior RNFL $(\beta=0.082, p<0.001)$ were related to increased VD excluding large vessels (Table 3 ).

\section{Macula Findings}

The direct comparison of macula parameters showed that control eyes exhibited higher superficial VD (control vs. PACS: 17.23 [12.01-20.94] vs. 13.80 [9.53-18.06], median [25th percentile to 75th percentile] $p=0.006$ ), and deep VD (control vs. PACS: 31.33 [24.42-36.18] vs. 27.92 [21.88-32.13], $p=0.004)$ compared to PACS eyes. In ad- 
Table 2. Comparison of optic disc parameters between control and PACS subjects

\begin{tabular}{llllll}
\hline & Parameter & Control & PACS & $p$ value & $\begin{array}{c}\text { Adjusted } \\
p \text { value* }\end{array}$ \\
& & & & 0.144 \\
\hline RNFL & Optic disc 4.5 mm peripheral & $111.90(104.02-120.36)$ & $115.58(106.74-126.31)$ & 0.048 \\
& GH segment S & $112.25(102.44-119.29)$ & $114.42(105.78-123.99)$ & 0.033 & 0.099 \\
& GH segment I & $112.51(104.38-122.33)$ & $115.70(107.20-126.61)$ & 0.061 & 0.183 \\
\hline VD including large vessels & Optic disc 4.5 mm peripheral & $56.40(54.58-57.72)$ & $57.11(54.98-58.73)$ & 0.049 & 0.147 \\
& GH segment S & $59.41(57.43-61.27)$ & $59.96(57.56-61.95)$ & 0.177 & 0.531 \\
& GH segment I & $58.94(56.43-60.96)$ & $59.45(57.44-61.52)$ & 0.128 & 0.348 \\
\hline VD excluding large vessels & Optic disc 4.5 mm peripheral & $49.81(48.16-51.14)$ & $50.23(48.17-51.54)$ & 0.240 & 0.72 \\
& GH segment S & $52.89(50.94-54.45)$ & $52.87(50.88-55.10)$ & 0.756 & 1 \\
& GH segment I & $52.79(50.90-54.85)$ & $53.29(51.48-54.98)$ & 0.486 & 1 \\
\hline
\end{tabular}

RNFL, retina nerve fiber layer; PACS, primary angle-closure suspect; GH segment S, Garway-Heath segment superior hemisphere; GH segment I, Garway-Heath segment inferior hemisphere;VD:vessel density. Data are expressed as median (25th percentile to 75 th percentile). * Adjusted $p$ values are $p$ values adjusted for 3 comparisons among each category using Bonferroni correction.

Table 3. Factors that influence optic disc VD excluding large vessels in all subjects

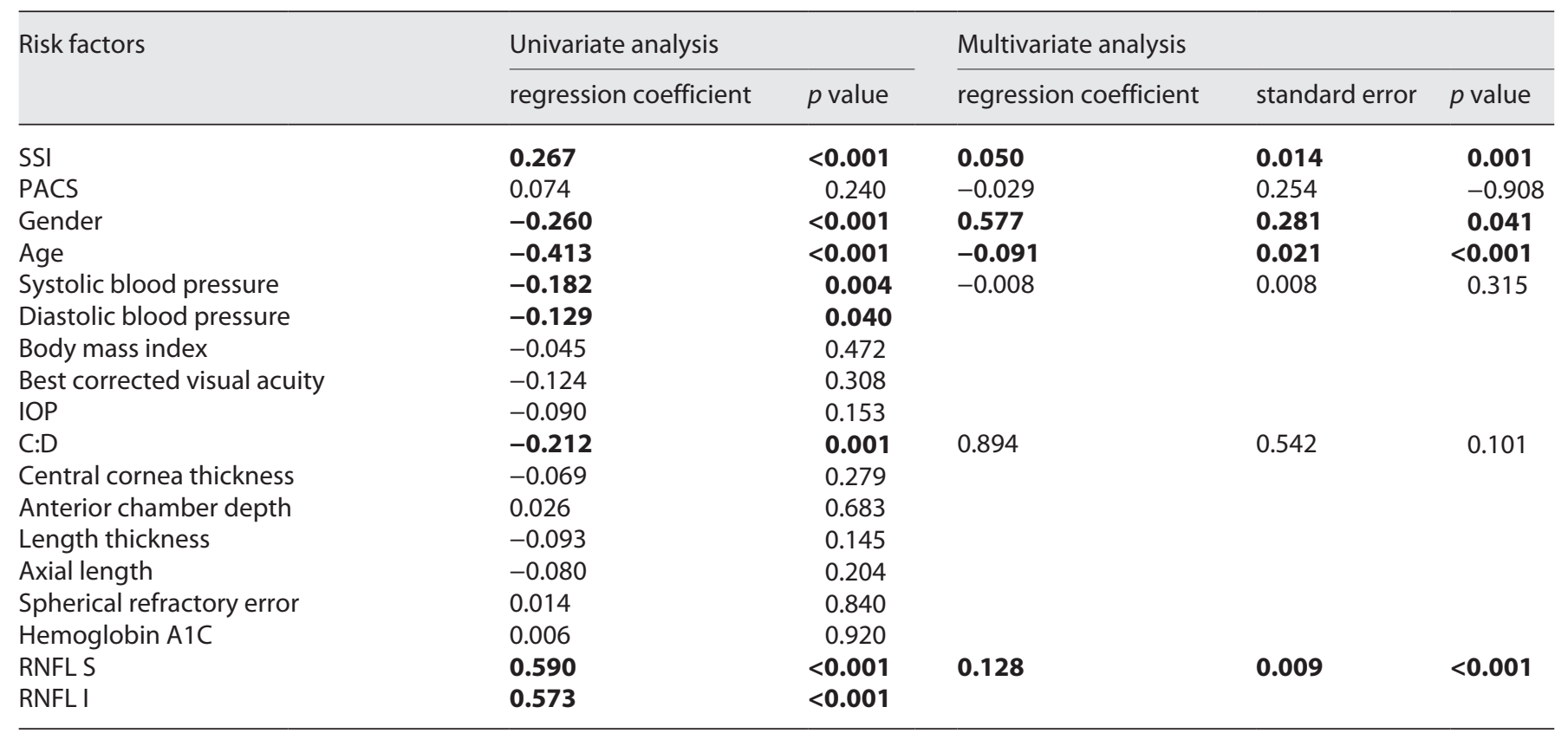

SSI, strength signal index; PACS, primary angle-closure suspect; RNFL S, retina nerve fiber layer superior hemisphere; RNFL I, retina nerve fiber layer inferior hemisphere; IOP, intraocular pressure; C:D, cup-to-disk ratio.

dition, control eyes showed smaller fovea avascular area (control vs. PACS: 0.35 [0.27-0.41] vs. 0.39 [0.31-0.46], $p$ $=0.006$ ) and shorter fovea avascular perimeter (control vs. PACS: 2.29 [2.01-2.59] vs. 2.45 [2.22-2.71], $p=0.004$ ) (Table 4). Representative patient's macula images are shown in Figure 2. We also compared macular parame- ters between the eyes with PACS and controls when axial lengths and lens thicknesses were matched (online suppl. Table; see www.karger.com/doi/10.1159/000520030 for all online suppl. material). The results were similar to data presented in Table 4. 
Table 4. Comparison of macula parameters between control and PACS subjects

\begin{tabular}{lllll}
\hline Parameter & Control & PACS & $p$ value & $\begin{array}{l}\text { Adjusted } \\
p \text { value* }\end{array}$ \\
\hline Macular superficial VD & $17.23(12.01-20.94)$ & $13.80(9.53-18.06)$ & 0.003 & $\mathbf{0 . 0 0 6}$ \\
Macular deep VD & $31.33(24.42-36.18)$ & $27.92(21.88-32.13)$ & 0.002 & $\mathbf{0 . 0 0 4}$ \\
FAZ area & $0.35(0.27-0.41)$ & $0.39(0.31-0.46)$ & 0.003 & $\mathbf{0 . 0 0 6}$ \\
PERIM & $2.29(2.01-2.59)$ & $2.45(2.22-2.71)$ & 0.002 & $\mathbf{0 . 0 0 4}$ \\
\hline
\end{tabular}

PACS, primary angle-closure suspect; VD, vessel density; FAZ, fovea avascular zone; PERIM, FAZ perimeter. Data are expressed as median (25th percentile to 75th percentile). * Adjusted $p$ values are $p$ values adjusted for 2 comparisons among each category using Bonferroni correction.

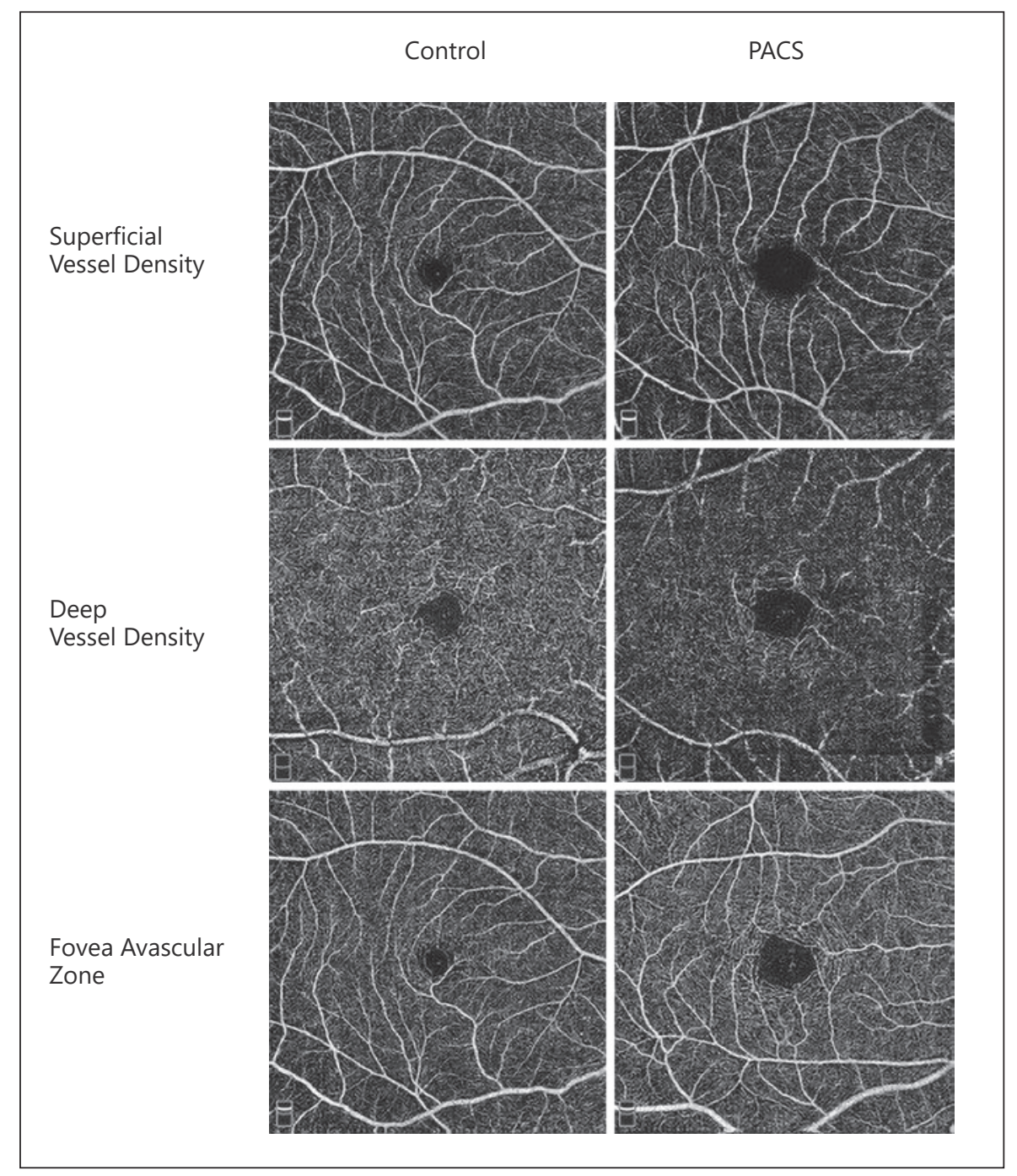

To study the potential factors that correlated with macula VD parameters, we performed multivariate analysis between all subjects and the 4 macula OCTA parameters (Table 5). Axial length and SSI are associated with macular vascular parameters. For example, longer axial length and larger SSI correlated with increased macular superficial and deep VD $(\beta=0.266, p<0.001$ and $\beta=$ $0.310, p<0.001$ for axial length, $\beta=1.709, p=0.004$ and 
Table 5. Multivariate regression between different factors and macular OCTA parameters in all subjects

\begin{tabular}{|c|c|c|c|c|}
\hline & Superficial VD & Deep VD & FAZ area & PERIM \\
\hline SSI & $0.236(<0.001)$ & $0.263(<0.001)$ & & \\
\hline PACS & $-0.106(0.095)$ & & $0.025(0.095)$ & $0.110(0.046)$ \\
\hline \multicolumn{5}{|l|}{ Gender } \\
\hline \multicolumn{5}{|l|}{ Age } \\
\hline \multicolumn{5}{|l|}{ Anterior chamber depth } \\
\hline Axial length & $0.266(<0.001)$ & $0.310(<0.001)$ & & \\
\hline
\end{tabular}

SSI, signal strength index; PACS, primary angle-closure suspect; FAZ, fovea avascular zone; VD, vessel density; PERIM, FAZ perimeter. Data are expressed as $\beta(p), \beta$; correlation coefficients, and $p ; p$ value in the multivariate analysis. The blank cells indicate data not included in multivariate analysis due to colinearity after lasso regression.

$\beta=2.557, p=0.001$ respectively). Interestingly, PACS was an independent variable associated with longer PERIM ( $\beta$ $=0.110, p=0.046)$ after adjusting age, gender, and axial length.

\section{Discussion}

Previous studies have shown that optic disc and macula parameter measurements are influenced by the magnification of the eye, imaging device, and the position of imaging device to the fundus structures [20]. In particular, shorter axial length is associated with thicker superior RNFL, larger FAZ area, and increased VD [21]. To address this issue, we employed Littmann's and Bennett's formulae to calculate the true size measurements. Specifically, we corrected for the magnification effect using the axial length, which was shown by Garway-Heath et al. [16] to be the most effective parameter that reduced the standard deviation of calculation error by $84-90 \%$. After the correction, our study showed that the PERIM remained statistically different between PACS eyes and normal eyes. This magnification effect adjustment was only performed for the actual lengths. No adjustments were needed for ratio measurements such as VD because vessel area and FAZ area are magnified by the same amount.

PACS eyes had similar RPC VD and RNFL compared to control eyes. Clinically, PACS eyes do not exhibit elevated IOP. However, it is unclear whether PACS patients experience increased diurnal IOP changes or undetected IOP spikes under certain conditions, such as a dark environment. OCTA enabled us to measure RPC, a unique plexus of capillary laying internally to RNFL. RPC also had fewer anastomosis compared to other vascular layers near the optic disc including the superior vascular plexus, the intermediate apical plexus, and the deep capillary plexus. This feature makes RPC particularly vulnerable to environmental assaults including IOP fluctuations [22]. Previous studies mainly focused on PAC and PACG. While most studies show thinning of RNFL and decrease of $\mathrm{VD}$ in PAC and PACG eyes, whether vasculature change precedes RNFL change remains controversial [23, 24]. Our finding of similar RPC between control and PACS supports that PACS subjects are similar to the general population in the optic disc in both RNFL and vascular structure.

Many studies have been performed on optic disc VD and age. Mansoori et al. [22] showed that RPC among 9to 63-year-old did not change with age. However, Pinhas et al. [25] found that the average RPC in healthy subjects significantly decreased with age in subjects 60 years or older. Since OCTA measures the movement of red blood cells, it effectively accounts for the actual blood supply to the tissue instead of the size of vessel itself [26-28]. We found a negative correlation between the VD and age. With aging, it is not surprising that the vessel walls become thicker and stiffer, and the vessel lumen becomes narrower. We also observed that the VD around optic nerve in men was less than in women. The same trend has been observed in early glaucoma patients [29]. The mechanism of this observation is unclear, although it might be a result of the vascular protective property of estrogen [30].

In our study, PACS eyes demonstrated a lower macula VD and larger FAZ. Without more precise measurements, it is difficult to say whether these changes affect visual function. Conflicting data exist in the literature regarding whether RNFL thinning and visual functional loss occur earlier than the changes in macular blood 
flow [ 31,32$]$. It is possible that macular vascular changes may represent early change of localized pathology. In this case, detecting these changes with OCTA may identify local vascular changes earlier than functional and structural changes. Abnormalities detected by OCTA may potentially precede PAS formation or RNFL/HVF changes. Longitudinal study is needed to examine whether these patients will develop clinically identified abnormalities in the future. Our study also shows PACS is an independent parameter associated with longer PERIM but not increased FAZ area. This can be explained by the higher number of irregular outlines. Since the same phenomena were observed in diabetic retinopathy and primary open-angle glaucoma, loss of FAZ circularity is commonly believed to be a good indicator of vascular dropout $[33,34]$.

While the explanation of abnormal OCTA macular blood flow in PACS eyes is still under investigation, in practice, our findings of abnormal macular vascularity indicate the importance of building a reference baseline for PACS patients. For example, if a PACS patient develops retinal vascular disease such as diabetic retinopathy, it is better to compare that patient's retinal vascular structure to otherwise healthy eyes with PACS than the general population.

The relationship of axial length and macula vasculature has been studied by OCTA. In general, an inverse relationship exists between axial length and superficial VD but not the FAZ area [21]. Some studies reported that the inverse relationships between axial length and superficial and deep VD in myopic eyes were not always explainable by retina expansion $[35,36]$. We are the first to report the relationship of axial length and macula vasculature in the PACS population. This may suggest metabolic changes in PACS eye compared to normal eyes.

Studies have showed the effect of SSI on VD measurement [18]. Czako et al. [19] found that SSI was positive related with VD values, while not with the non-flow area, which was identical to our results. This may be explained by that high-quality images help to distinguish different layers of the retinal structure and the vessel contour.

Our study has some limitations. First, the subjects underwent OCTA examination at different times of the day, and the data could potentially be influenced by diurnal fluctuation. Although VD might fluctuate throughout the day, no literature exists on the diurnal variation of healthy and PACS subjects [37]. Second, only Chinese patients were included in the study. Application of the results to other races or ethnicities needs to be cautious.

\section{Conclusion}

In summary, using OCTA, we did not find any differences in VD and RNFL between control and PACS eyes in the peripapillary region. However, PACS eyes showed lower superficial and deep VD as well as a larger FAZ. PACS is an independent variable associated with a longer PERIM.

\section{Acknowledgments}

The authors thank staff members from Zhangziying Hospital, Daxing District, Beijing, for their assistance with subject recruitment.

\section{Statement of Ethics}

The Ethical Review Board: the study was approved by the Ethics Committee of Clinical Research in Beijing Tongren Hospital, Capital Medical University. The approval number is TRECKY2019-071. Registration ID: the clinical registration number is ChiCTR2000037944 in Chinese Clinical Trial Registry. The informed consent to participate was signed by all participants attending this process.

\section{Conflict of Interest Statement}

The authors declare that they have no conflicts of interest.

\section{Funding Sources}

This study was funded by the National Natural Science Foundation of China (Grant No. 81970797).

\section{Author Contributions}

Ying Han and Shuning Li contributed to the conception of the study; Hui Feng, Yue Yang, Zhongyin Hu, Fancheng Yan, Mengya Zhao, Qian Liu, and Min Fu performed the experiment; Richard Lee provided some kind advice on the manuscript; Fei Xiang sorted out the data; Kai Cao performed the data analyses; Xiaohua Pan, Merry Z.C. Ruan, and Wudi Fan wrote the manuscript.

\section{Data Availability Statement}

The data was stored in the form of paper materials and the Epidata database, which was not disclosed. 


\section{References}

1 Radhakrishnan S, Chen PP, Junk AK, NouriMahdavi K, Chen TC. Laser peripheral iridotomy in primary angle closure: a report by the American Academy of Ophthalmology. Ophthalmology. 2018;125(7):1110-20.

2 Tham YC, LiX, Wong TY, Quigley HA, Aung T, Cheng CY. Global prevalence of glaucoma and projections of glaucoma burden through 2040: a systematic review and meta-analysis. Ophthalmology. 2014;121(11):2081-90.

3 Kavitha S, Zebardast N, Palaniswamy K, Wojciechowski R, Chan ES, Friedman DS, et al. Family history is a strong risk factor for prevalent angle closure in a South Indian population. Ophthalmology. 2014;121(11):2091-7.

4 Jiang Y, Chang DS, Zhu H, Khawaja AP, Aung $T$, Huang S, et al. Longitudinal changes of angle configuration in primary angle-closure suspects: the Zhongshan Angle-closure prevention trial. Ophthalmology. 2014;121(9):1699-705.

5 Chan KKW, Tang F, Tham CCY, Young AL, Cheung CY. Retinal vasculature in glaucoma: a review. BMJ Open Ophthalmol. 2017;1(1): e000032.

6 Chen CL, Bojikian KD, Gupta D, Wen JC, Zhang $\mathrm{Q}$, Xin C, et al. Optic nerve head perfusion in normal eyes and eyes with glaucoma using optical coherence tomography-based microangiography. Quant Imaging Med Surg. 2016;6(2): 125-33.

7 Chen CL, Bojikian KD, Wen JC, Zhang Q, Xin C, Mudumbai RC, et al. Peripapillary retinal nerve fiber layer vascular microcirculation in eyes with glaucoma and single-hemifield visual field loss. JAMA Ophthalmol. 2017;135(5):461-8.

8 Yarmohammadi A, Zangwill LM, Diniz-Filho A, Saunders LJ, Suh MH, Wu Z, et al. Peripapillary and macular vessel density in atients with glaucoma and single-hemifield visual field defect. Ophthalmology. 2017;124(5):709-19.

9 Zhao Q, Yang WL, Wang XN, Wang RK, You QS, Chu ZD, et al. Repeatability and reproducibility of quantitative assessment of the retinal microvasculature using optical coherence tomography angiography based on optical microangiography. Biomed Environ Sci. 2018;31(6): 407-12.

10 Fang D, Tang FY, Huang H, Cheung CY, Chen $\mathrm{H}$. Repeatability, interocular correlation and agreement of quantitative swept-source optical coherence tomography angiography macular metrics in healthy subjects. Br J Ophthalmol. 2019;103(3):415-20.

11 Yarmohammadi A, Zangwill LM, Diniz-Filho A, Suh MH, Yousefi S, Saunders LJ, et al. Relationship between optical coherence tomography angiography vessel density and severity of visual field loss in glaucoma. Ophthalmology. 2016; 123(12):2498-508.

12 Wang X, Jiang C, Ko T, Kong X, Yu X, Min W, et al. Correlation between optic disc perfusion and glaucomatous severity in patients with open-angle glaucoma: an optical coherence tomography angiography study. Graefes Arch Clin Exp Ophthalmol. 2015;253(9):1557-64.
13 Suh MH, Zangwill LM, Manalastas PI, Belghith A, Yarmohammadi A, Medeiros FA, et al. Deep retinal layer microvasculature dropout detected by the optical coherence tomography angiography in glaucoma. Ophthalmology.2016;123(12): 2509-18.

14 Atalay E, Nongpiur ME, Yap SC, Wong TT, Goh $\mathrm{D}$, Husain R, et al. Pattern of visual field loss in primary angle-closure glaucoma across different severity levels. Ophthalmology. 2016;123(9): 1957-64.

15 Dong J, Jia YD, Wu Q, Zhang S, Jia Y, Huang D, et al. Interchangeability and reliability of macular perfusion parameter measurements using optical coherence tomography angiography. $\mathrm{Br}$ J Ophthalmol. 2017;101(11):1542-9.

16 Garway-Heath DF, Rudnicka AR, Lowe T, Foster PJ, Fitzke FW, Hitchings RA. Measurement of optic disc size: equivalence of methods to correct for ocular magnification. Br J Ophthalmol. 1998;82(6):643-9.

17 Bennett AG, Rudnicka AR, Edgar DF. Improvements on Littmann's method of determining the size of retinal features by fundus photography. Graefes Arch Clin Exp Ophthalmol. 1994; 232(6):361-7.

18 Gao SS, Jia Y, Liu L, Zhang M, Takusagawa HL, Morrison JC, et al. Compensation for reflectance variation in vessel density quantification by optical coherence tomography angiography. Invest Ophthalmol Vis Sci. 2016;57(10):4485-92.

19 Czako C, Istvan L, Ecsedy M, Recsan Z, Sandor $\mathrm{G}$, Benyo F, et al. The effect of image quality on the reliability of OCT angiography measurements in patients with diabetes. Int $J$ Retina Vitreous. 2019;5:46.

20 Pach J, Pennell DO, Romano PE. Optic disc photogrammetry: magnification factors for eye position, centration, and ametropias, refractive and axial; and their application in the diagnosis of optic nerve hypoplasia. Ann Ophthalmol. 1989; 21(12):454-62.

21 Sampson DM, Gong P, An D, Menghini M, Hansen A, Mackey DA, et al. Axial length variation impacts on superficial retinal vessel density and foveal avascular zone area measurements using optical coherence tomography angiography. Invest Ophthalmol Vis Sci. 2017;58(7): 3065-72.

22 Mansoori T, Sivaswamy J, Gamalapati JS, Agraharam SG, Balakrishna N. Measurement of radial peripapillary capillary density in the normal human retina using optical coherence tomography angiography. J Glaucoma. 2017;26(3):241-

23 Mansoori T, Balakrishna N. Peripapillary vessel density and retinal nerve fiber layer thickness in patients with unilateral primary angle closure glaucoma with superior hemifield defect. J Curr Glaucoma Pract. 2019;13(1):21-7.

24 Wang X, Chen J, Kong X, Sun X. Immediate changes in peripapillary retinal vasculature after intraocular pressure elevation -an optical coherence tomography angiography study. Curr Eye Res. 2020;45(6):749-56.
25 Pinhas A, Linderman R, Mo S, Krawitz BD, Geyman LS, Carroll J, et al. A method for agematched OCT angiography deviation mapping in the assessment of disease- related changes to the radial peripapillary capillaries. PLoS One. 2018;13(5):e0197062.

26 Wong TY, Klein R, Klein BE, Meuer SM, Hubbard LD. Retinal vessel diameters and their associations with age and blood pressure. Invest Ophthalmol Vis Sci. 2003;44(11):4644-50.

27 Leung H, Wang JJ, Rochtchina E, Tan AG, Wong TY, Klein R, et al. Relationships between age, blood pressure, and retinal vessel diameters in an older population. Invest Ophthalmol Vis Sci. 2003;44(7):2900-4.

28 Kawasaki R, Wang JJ, Rochtchina E, Taylor B, Wong TY, Tominaga M, et al. Cardiovascular risk factors and retinal microvascular signs in an adult Japanese population: the Funagata Study. Ophthalmology. 2006;113(8):1378-84.

29 Wang S, Mendez-Hernandez C, Arribas-Pardo P, Salazar Quiñones L, Fernandez-Perez C, Garcia-Feijoo J. Gender-related influences on superficial papillary microcirculation measured with optical coherence tomography angiography in patients with glaucoma. Curr Eye Res. 2020;45: 1534-42.

30 Faria AF, de Souza MA, Geber S. Vascular resistance of central retinal artery is reduced in postmenopausal women after use of estrogen. Menopause. 2011;18(8):869-72.

31 Liu L, Edmunds B, Takusagawa HL, Tehrani S, Lombardi LH, Morrison JC, et al. Projection-resolved optical coherence tomography angiography of the peripapillary retina in glaucoma. Am J Ophthalmol. 2019;207:99-109.

32 Van Melkebeke L, Barbosa-Breda J, Huygens M Stalmans I. Optical coherence tomography angiography in glaucoma: a review. Ophthalmic Res. 2018;60(3):139-51.

33 Tam J, Dhamdhere KP, Tiruveedhula P, Manzanera S, Barez S, Bearse MA Jr, et al. Disruption of the retinal parafoveal capillary network in type 2 diabetes before the onset of diabetic retinopathy. Invest Ophthalmol Vis Sci. 2011;52(12): 9257-66.

34 Kwon J, Choi J, Shin JW, Lee J, Kook MS. Alterations of the foveal avascular zone measured by optical coherence tomography angiography in glaucoma patients with central visual field defects. Invest Ophthalmol Vis Sci. 2017;58(3): 1637-45.

35 Khan MH, Lam AKC, Armitage JA, Hanna L, To $\mathrm{CH}$, Gentle A. Impact of axial eye size on retinal microvasculature density in the macular region. J Clin Med. 2020;9(8):2539.

36 Fan H, Chen HY, Ma HJ, Chang Z, Yin HQ, Ng DS, et al. Reduced macular vascular density in myopic eyes. Chin Med J. 2017;130(4):445-51.

37 Mansouri K, Rao HL, Hoskens K, D’Alessandro E, Flores-Reyes EM, Mermoud A, et al. Diurnal variations of peripapillary and macular vessel density in glaucomatous eyes using optical coherence tomography angiography. J Glaucoma. 2018;27(4):336-41. 